\title{
Article \\ The Second Generalization of the Hausdorff Dimension Theorem for Random Fractals
}

\author{
Mohsen Soltanifar 1,2,3
}

1 Continuing Studies Division, Population Data BC, University of Victoria, B364-3800 Finnerty Road, Victoria, BC V8P 5C2, Canada; mohsensoltanifar@uvic.ca or mohsen.soltanifar@alum.utoronto.ca 2 Real World Analytics, Cytel Canada Health Inc., 802-777 West Broadway, Vancouver, BC V5Z 1J5, Canada

3 Biostatistics Division, Dalla Lana School of Public Health, University of Toronto, 620-155 College Street, Toronto, ON M5T 3M7, Canada

\begin{abstract}
In this paper, we present a second partial solution for the problem of cardinality calculation of the set of fractals for its subcategory of the random virtual ones. Consistent with the deterministic case, we show that for the given quantities of the Hausdorff dimension and the Lebesgue measure, there are aleph-two virtual random fractals with, almost surely, a Hausdorff dimension of a bivariate function of them and the expected Lebesgue measure equal to the latter one. The associated results for three other fractal dimensions are similar to the case given for the Hausdorff dimension. The problem remains unsolved in the case of non-Euclidean abstract fractal spaces.
\end{abstract}

Keywords: random fractals; fat fractal perculation; Hausdorff dimension; packing dimension; Assouad dimension; box dimension; existence; aleph-two

MSC: 28A80; 11K55; 03E10; 49J55

check for updates

Citation: Soltanifar, M. The Second Generalization of the Hausdorff Dimension Theorem for Random Fractals. Mathematics 2022, 10, 706. https://doi.org/10.3390/ math10050706

Academic Editors: Krzysztof Gdawiec and Agnieszka Lisowska

Received: 27 December 2021

Accepted: 22 February 2022

Published: 24 February 2022

Publisher's Note: MDPI stays neutral with regard to jurisdictional claims in published maps and institutional affiliations.

Copyright: (c) 2022 by the author. Licensee MDPI, Basel, Switzerland. This article is an open access article distributed under the terms and conditions of the Creative Commons Attribution (CC BY) license (https:// creativecommons.org/licenses/by/ $4.0 /)$.

\section{Introduction}

\subsection{Random Fractals}

Random fractals have emerged as the natural expansions of deterministic fractals, and their introductory mathematical treatment started with the works of Mandelbrot in the early 1970s [1,2]. Later, their rigorous mathematical treatment was solidified with the works of Taylor, Falconer and Graf in the mid-1980s [3-5]. These fractals use stochastic processes rather than deterministic processes in their constructions and are characterized by statistical self-similarity rather than the deterministic precise self-similarity. In detail, the small component parts of the fractal have the same probability distribution as the whole fractal. Some of their applications have emerged in financial markets [6], cosmogeny [7] and image synthesis [8]. As in their deterministic counterparts, random fractals are divided into two categories: the real random fractals and the virtual random fractals. Some examples of real-world random fractals include the human brain surface and the coastlines of the British Isles. Others, in the virtual random fractals category, include random Cantor sets, random von Koch curves and Galton-Watson fractals.

\subsection{Existence}

The existence of abstract mathematical objects and mathematical philosophy's importance has long been the subject of interest among mathematicians, and the first prominent argument for them roots in the work of Frege in the early 1950s [9]. Furthermore, it has long been discussed among mathematicians whether the existence of mathematical objects implies the possibility of their constructions and whether there is a systematic approach to the construction itself [10-12]. Furthermore, applications of constructive mathematical objects in many branches of mathematics and computer science add to the prominence of their investigations $[13,14]$. In the case of fractals, this prominence increases given their 
applications in many scientific fields, such as complex networks [15], electrical engineering [16], neuroscience [17], soil studies [18] and music [19]. Sets in fractal geometry are often characterized by their sizes measured by the fractal dimensions (e.g., Hausdorff dimension) and the space measures (e.g., Lebesgue measure). Hence, the investigation of the existence of fractals is formulated in terms of their fractal dimension and the associated space measure.

\subsection{Motivation}

Until the mid-2000s, literature in fractal geometry focused on calculating fractal dimensions and space measures of the discussed objects without a retrospective investigation of their existence for these two given quantities. The earliest treatment of the problem of retrospective existence of fractals in terms of their fractal dimension has been through the works of Sharapovs and Soltanifar [20,21]. The later work expanded the upon former and presented such existences as the Hausdorff dimension theorem (HDT) limited to deterministic, thin, virtual fractals with provisions of the cardinality of the continuum. Similar results to the HDT have been reported in the late 2010s in the works of Squillace and Gryszka [22,23]. The HDT has recently been generalized to deterministic fat virtual fractals with the provision of the cardinality of aleph-two coined as the generalized Hausdorff dimension theorem (GHDT) [24]. However, no information is available on the counterpart existence statement for the case of random fractals. Moreover, the current results in the deterministic realm are limited to the Hausdorff dimension, and their validity is unclear for other fractal dimensions. Finally, there are still open questions about whether the cardinality of the set of fractals depends on their deterministic or random nature as well as the applied fractal dimension in counting their set [24].

\subsection{Study Outline}

This work offers a counterpart to the existing result for random virtual fractals for the given fractal dimension and the expected Lebesgue measure in n-dimensional Euclidean spaces. It also provides an answer to the above-posed questions regarding independency of the cardinality of the set of virtual fractals and their deterministic or random status and the applied fractal dimensions. The work outline is as follows: First, it introduces the fat fractal percolations (FFP) and summarizes some of their topological properties and fractal dimensions. Second, the ancillary proofs are furnished for establishing these properties in the following section. Third, it establishes two key lemmas regarding the cardinality of the power set of the surviving FFP and the case of HDT for random fractals. Finally, using the two mentioned lemmas, it establishes the random counterpart of the GHDT coined as the second generalized Hausdorff dimension theorem (SGHDT).

\section{Preliminaries}

The reader who has studied random fractals is well-equipped with the following definitions and key properties of the fat fractal percolation (FFP). The summary of definitions and some key properties of the Hausdorff dimension and the topological dimension (denoted by $\operatorname{dim}_{H}(),. \operatorname{dim}_{\text {ind }}($.$) , respectively) are presented by Soltanifar [24]. Furthermore,$ the summary and key properties of the packing dimension, Assouad dimension and the boxing dimension (denoted by $\operatorname{dim}_{P}(),. \operatorname{dim}_{A}(),. \operatorname{dim}_{B}($.$) , respectively) are presented by$ Chen $[25,26]$. Henceforth, in this paper, we consider the n-dimensional unit cube $I_{n}(n \geq 1)$ in the Euclidean space $\mathbb{R}^{n}$ with its conventional Euclidean metric and the Lebesgue measure of $\lambda_{n}($.$) .$

The earliest ideas of the FFP root to Mandelbrot's work in 1982 [27]. Later, their geometry for the case of random Cantor fractals was discussed in detail by Falconer and Grimmett [28]. The construction process of a version of the FFP is as follows: Given a dimension $n \geq 1$, an index parameter $m \geq 2$ and a non-decreasing sequence $\vec{p}=\left\{p_{k}\right\}_{k=1}^{\infty}$ in $(0,1]$, let $C_{n, m}, \vec{p}(0)$ be the unit cube $I_{n}$. Divide $C_{n, m}, \vec{p}(0)$ into $m^{n}$ equal closed subcubes, each with length $m^{-1}$, and, then select each subcube independently with probability $p_{1}$. 
Denote the union of chosen subcubes as $C_{n, m}, \vec{p}(1)$. Continuing this process, at the stage $k>1$, divide each subcube of $C_{n, m, \vec{p}}(k-1)$ into $m^{n}$ equal closed subcubes, each with length $m^{-k}$, and, then select each subcube independently with probability $p_{k}$. Similar to the case $k=1$, denote the union of chosen subcubes as $C_{n, m}, \vec{p}(k)$. The sequence of random closed sets $\left\{C_{n, m, \vec{p}}(k)\right\}_{k=0}^{\infty}$ is decreasing.

Definition 1. The fat fractal percolation(FFP) $C_{n, m, \vec{p}}$ associated with dimension $n \geq 1$, index parameter $m \geq 2$ and the non-decreasing sequence $\vec{p}$ is defined as:

$$
C_{n, m, \vec{p}}=\bigcap_{k=0}^{\infty} C_{n, m, \vec{p}}(k)
$$

To study FFP in Definition 1, we consider the probability space $\left(\Omega_{n}, \mathcal{F}_{n}, P_{p}\right),(n \geq 1)$ with the following characteristics: Let $\Omega_{n}=I_{n}$, and $\mathcal{F}_{n}(n \geq 1)$ be its space of compact subsets. We define a natural product probability measure $P_{p}$ as follows: let $D_{n, m, k}(k \geq 0)$ be the set of all $\left(m^{n}\right)^{k}$ closed subcubes of $I_{n}$ each with side length $m^{-k}$. For any $k \geq 1$ and each $E \in D_{n, m, k}$ define a probaility measure $P_{n, m, \vec{p}}(E)=\frac{1_{\mathcal{P}\left(c_{n, m}, \vec{p}^{(k)}\right)}(E)}{\prod_{l=1}^{k} p_{l} \times\left(m^{n}\right)^{k}}$, where $\mathcal{P}($.$) refers$ to the power set. Then, by the extension theorem [29] there is a unique measure $P_{p}$ on $I_{n}$ such that $P_{p}(E)=P_{n, m, \vec{p}}(E)$, for all $E \in D_{n, m, k}, k \geq 1$. We say a property holds almost surely (a.s.) when it holds on a set of full $P_{p}$ measures.

Remark 1. The probability space $\left(\Omega_{n}, \mathcal{F}_{n}, P_{p}\right),(n \geq 1)$ can be easily generalized for non-unit $n$ dimensional cubes. To see this, let $0 \leq a<b$, and $I_{n, a, b}=[a, b]^{n}$. Define $\Omega_{n, a, b}=(b-a) \Omega_{n}+a$, $\mathcal{F}_{n, a, b}=\left\{(b-a) E+a \mid E \in \mathcal{F}_{n}\right\}$, and $P_{p, a, b}$ by $P_{p, a, b}(E)=P_{p}\left(\frac{E-a}{b-a}\right),\left(E \subseteq I_{n, a, b}\right)$. Then, $\left(\Omega_{n, a, b}, \mathcal{F}_{n, a, b}, P_{p, a, b}\right)$ is a probability space. Trivially, $\left(\Omega_{n}, \mathcal{F}_{n}, P_{p}\right)=\left(\Omega_{n, 0,1}, \mathcal{F}_{n, 0,1}, P_{p, 0,1}\right),(n \geq 1)$. In the upcoming results, we will use this probability space whenever it is useful within its context.

Remark 2. We refer to the set of random fractals in $\mathbb{R}^{n}$ via random fractals characterized by the probability space $\left(\Omega_{n, a, b}, \mathcal{F}_{n, a, b}, P_{p, a, b}\right)$ where $-\infty<a<b<\infty$ are clear from the context of the discussion.

Remark 3. Given $b>0$ and $n \geq 1$. Then, the two probability spaces $\left(\Omega_{n, 0, b}, \mathcal{F}_{n, 0, b}, P_{p, 0, b}\right)$, and $\left(\Omega_{n, b, 2 b}, \mathcal{F}_{n, b, 2 b}, P_{p, b, 2 b}\right)$ can be considered as two "subspaces" of the probability space $\left(\Omega_{n, 0,2 b}\right.$, $\left.\mathcal{F}_{n, 0,2 b}, P_{p, 0,2 b}\right)$, in the set theoric context. To see this, it is sufficient to consider that in the first round of the above construction process, all chosen subcubes from $\Omega_{n, 0,2 b}$ are to be chosen from either $\Omega_{n, 0, b}$ or $\Omega_{n, b, 2 b}$, respectively.

Remark 4. Given random fractal $C$ in $\mathbb{R}^{n}$, both of its Lebesgue measure $\lambda_{n}(C)$ and fractal dimension $\operatorname{dim}_{F}(C)$ are random variables. Consistent with the literature on random fractals, we characterize $C$ in terms of its expected Lebesgue measure and its almost sure value of the fractal dimension.

We are interested in the crucial topological and geometrical characteristics of the above-defined FFPs (see the next section for proofs). The first question is when the FFP survives the extinction (i.e., $C_{n, m}, \vec{p} \neq \varnothing$ )? The first theorem discusses this question and sheds light on some of its topological properties [30-33]:

Theorem 1. Let $C_{n, m, \vec{p}}$ be the FFP constructed as in Definition 1. Then, for $\alpha=\varliminf_{k \rightarrow \infty}\left(\prod_{l=1}^{k} p_{l}\right)^{\frac{1}{k}}$ and $\beta=\prod_{k=1}^{\infty} p_{k}^{m^{n k}}$ we have:

(i) $C_{n, m, \vec{p}}$ is an empty set or a non-empty set with positive probability whenever $\alpha \leq m^{-n}$, or $\alpha>m^{-n}$, respectively. Moreover, in the later condition, it is uncountable as well.

(ii) $C_{n, m, \vec{p}}$ has an empty interior or non-empty interior whenever $\beta=0$ or $\beta>0$, respectively. Furthermore, in the later condition, it equals almost surely to a union of finitely many cubes. 
Next, the most two prominent quantities essential for our upcoming results are the Fractal dimension and the Lebesgue measure. We refer to the fractal dimension (denoted by $\left.\operatorname{dim}_{F}().\right)$ as one of four dimesnions: the Hausdorff dimension, the packing dimension [34], the Assouad dimension [35,36] or the box dimension [37]. The following theorem quantifies these key quantities [25,31].

Theorem 2. Let $C_{n, m}, \vec{p}$ be the FFP constructed as in Definition 1. Then:

(i) $E\left(\lambda_{n}\left(C_{n, m, \vec{p}}\right)\right)=\prod_{k=1}^{\infty} p_{k}$

(ii) $\operatorname{dim}_{H}\left(C_{n, m, \vec{p}}\right) \stackrel{\text { a.s. }}{=} n+\log _{m}\left(\varliminf_{k \rightarrow \infty}\left(\prod_{l=1}^{k} p_{l}\right)^{\frac{1}{k}}\right)$,

(iii) $\operatorname{dim}_{P}\left(C_{n, m, \vec{p}}\right) \stackrel{\text { a.s. }}{=} \varlimsup_{k \rightarrow \infty}\left(\frac{n+\log _{m}\left(\prod_{l=1}^{k+1} p_{l}\right)^{\frac{1}{k+1}}}{1+\frac{1}{n} \log _{m}\left(p_{k+1}^{\frac{1}{k+1}}\right)}\right)$,

(iv) $\operatorname{dim}_{A}\left(C_{n, m, \vec{p}}\right) \stackrel{\text { a.s. }}{=} n+\varlimsup_{t \rightarrow \infty}\left(\sup _{k \geq 1}\left(\log _{m}\left(\prod_{l=k}^{t+k} p_{l}\right)^{\frac{1}{t+1}}\right)\right)$,

(v) $\operatorname{dim}_{B}^{\text {lower }}\left(C_{n, m, \vec{p}}\right)=\operatorname{dim}_{H}\left(C_{n, m, \vec{p}}\right)$ and $\operatorname{dim}_{B}^{\text {upper }}\left(C_{n, m, \vec{p}}\right)=\operatorname{dim}_{P}\left(C_{n, m}, \vec{p}\right)$.

An immediate consequence of Theorem 2 is the following:

Corollary 1. The FFP constructed in Definition 1 has a positive expected Lebesgue measure if and only if its Hausdorff dimension equals to $n$, almost surely.

We finish this section with some prominent examples useful for the key results in the subsequent section.

Example 1. Let the sequence of probabilities $\left\{p_{k}\right\}_{k=1}^{\infty}$ be defined by $p_{k}=p^{a_{k}}$ where $m^{-n}<p<1$ is fixed and $a_{k}>0(k \geq 1)$. Then, by above theorem, it follows that:

(i) $E\left(\lambda_{n}\left(C_{n, m, \vec{p}}\right)\right)=p^{\sum_{k=1}^{\infty} a_{k}}$,

(ii) $\operatorname{dim}_{H}\left(C_{n, m, \vec{p}}\right) \stackrel{\text { a.s. }}{=} n+\left(\underline{\lim }_{k \rightarrow \infty} \frac{\sum_{l=1}^{k} a_{l}}{k}\right) \times \log _{m}(p)$,

(iii) $\operatorname{dim}_{P}\left(C_{n, m, \vec{p}}\right) \stackrel{\text { a.s. }}{=} \varlimsup_{k \rightarrow \infty}\left(\frac{n+\frac{\sum_{l 1}^{k+1} a_{l}}{k+1} \times \log _{m}(p)}{1+\frac{k_{k+1}}{k+1} \frac{1}{n} \log _{m}(p)}\right)$,

(iv) $\operatorname{dim}_{A}\left(C_{n, m, \vec{p}}\right) \stackrel{\text { a.s. }}{=} n+\varlimsup_{t \rightarrow \infty}\left(\sup _{k \geq 1} \frac{\sum_{l=k}^{k+t} a_{l}}{t+1} \times \log _{m}(p)\right)$.

We will use this special example for the proof of Lemma 2 in the subsequent section.

Example 2. Let, in Example 1, $a_{k}=1(k \geq 1)$. Then, the obtained random fractals are referred to as Mandelbrot fractal percolations (MFP). In particular, for $p>m^{-n}$ the MFP survives extinction, $E\left(\lambda_{n}\left(C_{n, m}, \vec{p}\right)\right)=0$, and $\operatorname{dim}_{H}\left(C_{n, m}, \vec{p}\right)=\operatorname{dim}_{P}\left(C_{n, m}, \vec{p}\right)=\operatorname{dim}_{A}\left(C_{n, m}, \vec{p}\right)=$ $\operatorname{dim}_{B}\left(C_{n, m, \vec{p}}\right)=n+\log _{m}(p)$, almost surely. We write for simplicity $\operatorname{dim}_{F}\left(C_{n, m}, \vec{p}\right)=n+$ $\log _{m}(p)$, almost surely.

\section{Some Ancillary Proofs}

This section provides the proofs for the above results.

\subsection{Proof for Theorem 1: (i)}

This is the immediate consequence of Theorem 2 (ii) and the properties of the Hausdorff dimension [30]. 


\subsection{Proof for Theorem 2: (i)-(v)}

(i) Let $k \geq 1$ be fixed. Then, by conditioning and mathematical induction, the number of subcubes $X_{k}$ in $C_{n, m}, \vec{p}(k)$ satisfies $X_{k} \sim \operatorname{Binomial}\left(\left(m^{n}\right)^{k}, \prod_{l=1}^{k} p_{l}\right)$. However, each subcube has length $m^{-k}$ and Lebesgue measure of $\left(m^{n}\right)^{-k}$. Hence,

$$
E\left(\lambda_{n}\left(C_{n, m, \vec{p}}(k)\right)\right)=E\left(X_{k} \cdot\left(m^{n}\right)^{-k}\right)=\prod_{l=1}^{k} p_{l},(k \geq 1) .
$$

Now, the result follows from an application of the Fubini's Theorem and letting $k \rightarrow+\infty$.

(ii) Let $d=n, M_{k}=m$ and $N_{k}=p_{k} * m^{n}(k \geq 1)$ as in $[25,26]$. Then:

$$
\begin{aligned}
\operatorname{dim}_{H}\left(C_{n, m, \vec{p}}\right) & =\varliminf_{k \rightarrow \infty} \frac{\log \left(\prod_{l=1}^{k} N_{l}\right)}{-\log \left(\prod_{l=1}^{k} M_{l}^{-1}\right)}=\varliminf_{k \rightarrow \infty} \frac{\log \left(\left(\prod_{l=1}^{k} p_{l}\right)\left(m^{k}\right)^{n}\right)}{\log \left(m^{k}\right)} \\
& =\varliminf_{k \rightarrow \infty}\left(n+\frac{\log \left(\prod_{l=1}^{k} p_{l}\right)}{\log \left(m^{k}\right)}\right)=\varliminf_{k \rightarrow \infty}\left(n+\frac{\log \left(\left(\prod_{l=1}^{k} p_{l}\right)^{\frac{1}{k}}\right)}{\log (m)}\right) \\
& =n+\varliminf_{k \rightarrow \infty} \log _{m}\left(\left(\prod_{l=1}^{k} p_{l}\right)^{\frac{1}{k}}\right)=n+\log _{m}\left(\varliminf_{k \rightarrow \infty}\left(\prod_{l=1}^{k} p_{l}\right)^{\frac{1}{k}}\right) .
\end{aligned}
$$

(iii) Similar to the case for Hausdorff dimension, let $d=n, M_{k}=m$ and $N_{k}=p_{k} * m^{n}(k \geq 1)$ as in $[25,26]$. Then:

$$
\begin{aligned}
\operatorname{dim}_{P}\left(C_{n, m, \vec{p}}\right) & =\varlimsup_{k \rightarrow \infty} \frac{\log \left(\prod_{l=1}^{k+1} N_{l}\right)}{-\log \left(\prod_{l=1}^{k} M_{l}^{-1}\right)+\frac{1}{n} \log \left(N_{k+1}\right)}=\varlimsup_{k \rightarrow \infty}\left(\frac{\log \left(\left(\prod_{l=1}^{k+1} p_{l}\right)\left(m^{n(k+1)}\right)\right)}{\log \left(m^{k}\right)+\frac{1}{n} \log \left(p_{k+1} m^{n}\right)}\right) \\
& =\varlimsup_{k \rightarrow \infty}\left(\frac{n(k+1) \log (m)+\log \left(\prod_{l=1}^{k+1} p_{l}\right)}{(k+1) \log (m)+\frac{1}{n} \log \left(p_{k+1}\right)}\right)=\varlimsup_{k \rightarrow \infty}\left(\frac{n+\frac{\log \left(\prod_{l=1}^{k+1} p_{l}\right)}{(k+1) \log (m)}}{1+\frac{\log \left(p_{k+1}\right)}{n(k+1) \log (m)}}\right) \\
& =\varlimsup_{k \rightarrow \infty}\left(\frac{n+\log _{m}\left(\prod_{l=1}^{k+1} p_{l}\right)^{\frac{1}{k+1}}}{1+\frac{1}{n} \log _{m}\left(p_{k+1}^{\frac{1}{k+1}}\right)}\right) .
\end{aligned}
$$

(iv) Similar to the case for the Hausdorff dimension, let $d=n, M_{k}=m$ and $N_{k}=$ $p_{k} * m^{n}(k \geq 1)$ as in $[25,26]$. Then:

$$
\begin{aligned}
\operatorname{dim}_{A}\left(C_{n, m, \vec{p}}\right) & =\varlimsup_{t \rightarrow \infty}\left(\sup _{k \geq 1}\left(\frac{\prod_{l=k}^{k+t} N_{l}}{-\log \left(\prod_{l=k}^{k+t} M_{l}^{-1}\right)}\right)=\varlimsup_{t \rightarrow \infty}\left(\sup _{k \geq 1} \frac{\log \left(\prod_{l=k}^{k+t}\left(p_{l} \cdot m^{n}\right)\right)}{\log \left(\prod_{l=k}^{k+t} m\right)}\right)\right. \\
& =\varlimsup_{t \rightarrow \infty}\left(\sup _{k \geq 1} \frac{\log \left(\left(\prod_{l=k}^{k+t} p_{l}\right) \cdot\left(m^{n(t+1)}\right)\right)}{\log \left(m^{t+1}\right)}\right) \\
& =\varlimsup_{t \rightarrow \infty}\left(\sup _{k \geq 1} \frac{\log \left(\prod_{l=k}^{k+t} p_{l}\right)+n \log \left(m^{t+1}\right)}{\log \left(m^{t+1}\right)}\right)=\varlimsup_{t \rightarrow \infty}\left(\sup _{k \geq 1} \frac{\log \left(\prod_{l=k}^{k+t} p_{l}\right)}{(t+1) \log (m)}+n\right) \\
& =\varlimsup_{t \rightarrow \infty}\left(\sup _{k \geq 1} \log _{m}\left(\left(\prod_{l=k}^{k+t} p_{l}\right)^{\frac{1}{t+1}}\right)+n\right)=n+\varlimsup_{t \rightarrow \infty}\left(\sup _{k \geq 1}\left(\log _{m}\left(\prod_{l=k}^{t+k} p_{l}\right)^{\frac{1}{t+1}}\right)\right)
\end{aligned}
$$

(iv) This is the direct consequence of [25].

\subsection{Proof for Corollary 1}

It is sufficient to use the representation $l=\prod_{k=1}^{\infty} p_{k}$. First, assume $l>0$. Then, using the Calculus Theorem on the limit of composition of continuous functions and the 
representation in part (ii), the sufficiency is proved. Second, assume $l=0$. Then, using the $\epsilon-\delta$ definition for the sequences and taking $\epsilon=\frac{1}{2}$, the necessity is proved.

\section{Main Results}

We generalize the existential generalized Hausdorff dimension theorem (GHDT) from deterministic fractals and one fractal dimension to random fractals and four fractal dimensions. The existential cardinality is aleph-two, as well. The construction process is accomplished in three stages: (i) calculting the cardinality of the power set of a surviving random fractal; (ii) showing the existence of a continuum of random fractals with a plausible fractal dimension and expected Lebesgue measure in $n$-dimensional Euclidean space $\mathbb{R}^{n}(n \geq 1)$ and (iii) generalizing the result in the second stage to the cardinal of aleph-two. We begin with the following Lemma of cardinality calculation, which plays a key role in the SGHDT.

Lemma 1. Given a random fractal $C_{n, m}, \vec{p}$ with almost surely positive Hausdorff dimension in the unit cube $I^{n}(n \geq 1)$. Then, the cardinality of its power set equals aleph-two.

Proof. Any countable event has a Hausdorff dimension of zero, almost surely [38]. Hence, any event with almost surely positive Hausdorff dimension is uncountable. Accordingly, its power set has cardinality of aleph-two given the generalized continuum hypothesis $(\mathrm{GCH})$.

Next, equipped with preliminary results in the previous section, we establish our first major result on the existence of random fractals for the given fractal dimension and Lebesgue measure. This result on its own is a direct generalization of the Hausdorff dimension theorem (HDT) [21] from the case of deterministic thin fractals to the case of random fat fractals, and, from one fractal dimension to four fractal dimensions.

Lemma 2. For any real $r>0$ and $l \geq 0$ there are continuum random fractals with the Hausdorff dimension $r .1_{\{0\}}(l)+n .1_{(0, \infty)}(l)$, almost surely and the expected Lebesgue measure $l$ in $\mathbb{R}^{n}(\lceil r\rceil \leq n)$.

Proof. We consider two sets of random fractals $C_{n, m, \vec{p}_{a}}$ where each has cardinality of continuum and its members survive extinction. Table 1 summarizes the key features of these two sets by applying Example 1.

Table 1. Two sets with cardinality of continuum of random fractals in $\mathbb{R}^{n}(n \geq 1)$.

\begin{tabular}{lcccc}
\hline$C_{\boldsymbol{n}, \boldsymbol{m}, \vec{p}_{a}}$ & $\vec{p}_{a}=\left\{p_{\boldsymbol{k}}(a)\right\}_{k=\mathbf{1}}^{\infty}$ & $E(\lambda())$. & $\operatorname{dim}_{\boldsymbol{H}}()$. & Constraints \\
\hline$\# 1$ & $p_{k}(a)=p^{a 1_{\{1\}}(k)+1_{[2,+\infty)}(k)}$ & 0 & $n+\log _{m}(p)$ & $a \geq 1$ \\
$\# 2$ & $p_{k}(a)=p^{\left(a^{k-1}-a^{k}\right)}$ & $p$ & $n$ & $0<a<1$ \\
\hline
\end{tabular}

Notes: $m^{-n}<p<1$ is taken as fixed. Values for the Hausdorff dimension are taken almost surely. Each constraint has the cardinality of continuum for the parameter $a$.

Now, given plausible values for the Hausdorff dimension and the Lebesgue measure we have three major cases:

(i) $0<r \notin \mathbb{N}, l=0$ :

Here, for $n \geq\lfloor r\rfloor+1=\lceil r\rceil$, and $p=m^{r-n}$ for the sets \#1 in Table 1 it follows that $\operatorname{dim}_{H}\left(C_{n, m, \vec{p}_{a}}\right)=r$, almost surely.

(ii) $0<r \in \mathbb{N}, l=0$ :

Here, for $n \geq r=\lceil r\rceil$, by part (i) for the sequence $\left\{r-2^{-k}\right\}_{k=1}^{\infty}$ there is corresponding sequence $\left\{C_{n, m, \vec{p}_{a}}^{(k)}\right\}_{k=1}^{\infty}$ such that $\operatorname{dim}_{H}\left(C_{n, m, \vec{p}_{a}}^{(k)}\right)=r-2^{-k},(k \geq 1)$, and $E\left(\lambda_{n}\left(C_{n, m, \vec{p}_{a}}^{(k)}\right)\right)=0$, $(k \geq 1)$. Next, take $C=\cup_{k=1}^{\infty} C_{n, m, \vec{p}_{a}}^{(k)}$, then $\operatorname{dim}_{H}(C)=\sup _{1 \leq k \leq \infty}\left(\operatorname{dim}_{H}\left(C_{n, m, \vec{p}_{a}}^{(k)}\right)\right)=r$, 
and $E\left(\lambda_{n}(C)\right)=0$. Finally, the assertion follows from the uncountability of the sets in part (i).

(iii) $0<r \in \mathbb{N}, l>0$ :

Here, for $n \geq r=\lceil r\rceil$, and $p=\frac{l}{\lfloor l\rfloor+1}$ for the sets \#2 in Table 1 it follows that $\operatorname{dim}_{H}\left((\lfloor l\rfloor+1)^{\frac{1}{n}} * C_{n, m, \vec{p}_{a}}\right)=r$, almost surely, and $E\left(\lambda_{n}\left((\lfloor l\rfloor+1)^{\frac{1}{n}} * C_{n, m, \vec{p}_{a}}\right)\right)=l$.

Finally, we complete the proof by considering the fact that constructed random fractals in cases (i), (ii) belong to the standard probability space $\left(\Omega_{n, 0,1}, \mathcal{F}_{n, 0,1}, P_{p, 0,1}\right)$ while those in case (iii) belong to the generalized probability space $\left(\Omega_{n, a, b}, \mathcal{F}_{n, a, b}, P_{p, a, b}\right): a=0$, $b=(\lfloor l\rfloor+1)^{\frac{1}{n}}$.

Remark 5. We can prove Lemma 2 in an alternative method. In this method, set $a=1$ and $a=\frac{1}{2}$ for the sets of random fractals in Table 1 , respectively. Then, consider the set $\mathcal{G}_{n}$ with the cardinality of a continuum of random fractals defined by $\mathcal{G}_{n}=\left\{\cup_{k \in I}\left(2^{-k} C_{n, m}, \vec{p}_{a}+\left(1-2^{k-1}\right) 1_{n}\right) \mid I \subseteq\right.$ $\mathbb{N}$ is infinite, $\left.1_{n}=\sum_{i=1}^{n} e_{i}(n \geq 1)\right\}$. Finally, the new proof is completed by considering a similar argument to the outlined three major cases mentioned in the first proof of the Lemma 2.

The constructed random fractals in Lemma 2 share one key feature with the deterministic ones in [24]: there is a continuum of the random fractals with the same fractal dimension and the same expected Lebesgue measure in the Euclidean spaces $\mathbb{R}^{n+1}-\mathbb{R}^{n}(n \geq 1)$ as those in $\mathbb{R}^{n}(n \geq 1)$. This is a direct consequence of considering $\Omega_{n, a, b}$ isomorphic to the subspace of $\Omega_{n, a, b} \times\{0\} \subseteq \Omega_{n+1, a, b}$, for the contextual $a<b$. Furthermore, as in the deterministic case, the result in Lemma 2 is limited to providing the highest possible cardinal number of aleph-two. Our final result provides them.

Theorem 3 (Second Generalized Hausdorff Dimension Theorem). For any real $r>0$ and $l \geq 0$, there are aleph-two random fractals with the Hausdorff dimension $r .1_{\{0\}}(l)+n \cdot 1_{(0, \infty)}(l)$ almost surely, and expected Lebesgue measure lin $\mathbb{R}^{n}$ where $(\lceil r\rceil \leq n)$.

Proof. Let $r>0, l \geq 0$ and fix $n \geq\lceil r\rceil$. Then, by two applications of Lemma 2, we have the following families of random fractals where each has the minimum cardinality of the continuum:

$$
\begin{aligned}
& \mathcal{G}_{1}(r, l)=\left\{G \in \mathcal{F}_{n,(\lfloor l\rfloor+1)^{\frac{1}{n}}, 2(\lfloor l\rfloor+1)^{\frac{1}{n}}} \mid \operatorname{dim}_{H}(G) \stackrel{\text { a.s. }}{\leq} \frac{r}{2}, E\left(\lambda_{n}(G)\right)=0\right\} \\
& \mathcal{G}_{2}(r, l)=\left\{G \in \mathcal{F}_{n, 0,(\lfloor l\rfloor+1)^{\frac{1}{n}}} \mid \operatorname{dim}_{H}(G) \stackrel{\text { a.s. }}{=} r .1_{\{0\}}(l)+n .1_{(0, \infty)}(l), E\left(\lambda_{n}(G)\right)=l\right\} .
\end{aligned}
$$

Next, by Remark 2, both families of events in Equation (2) belong to the probability space $\left(\Omega_{n, 0,2 b}, \mathcal{F}_{n, 0,2 b}, P_{p, 0,2 b}\right)$ where $b=(\lfloor l\rfloor+1)^{\frac{1}{n}}$. Furthermore, an application of Lemma 1 showes that the first family $\mathcal{G}_{1}(r, l)$ in Equation (2) has cardinality of aleph-two. Consequently, the following family of random fractals has cardinality of aleph-two:

$$
\mathcal{G}(r, l)=\left\{G_{1} \cup G_{2} \mid G_{1} \in \mathcal{G}_{1}(r, l), G_{2} \in \mathcal{G}_{2}(r, l)\right\} .
$$

Furthermore, let $G \in \mathcal{G}(r, l)$. Then, by definition, there are $G_{i} \in \mathcal{G}_{i}(i=1,2)$ such that: $G=G_{1} \cup G_{2}$. Now, by conventional properties of the Hausdorff dimension:

$$
\begin{aligned}
\operatorname{dim}_{H}(G) & =\max \left(\operatorname{dim}_{H}\left(G_{1}\right), \operatorname{dim}_{H}\left(G_{2}\right)\right) \\
& =\operatorname{dim}_{H}\left(G_{2}\right) \stackrel{\text { a.s. }}{=} r .1_{\{0\}}(l)+n \cdot 1_{(0, \infty)}(l) .
\end{aligned}
$$


Furthermore, we have:

$$
\begin{aligned}
E\left(\lambda_{n}(G)\right) & =E\left(\lambda_{n}\left(G_{1} \cup G_{2}\right)\right) \geq E\left(\lambda_{n}\left(G_{2}\right)\right)=l \\
& =E\left(\lambda_{n}\left(G_{1}\right)\right)+E\left(\lambda_{n}\left(G_{2}\right)\right) \geq E\left(\lambda_{n}\left(G_{1} \cup G_{2}\right)\right)=E\left(\lambda_{n}(G)\right)
\end{aligned}
$$

yielding:

$$
E\left(\lambda_{n}(G)\right)=l .
$$

This completes the proof.

Remark 6. Using Theorem 2 and Example 1, the results in Lemma 2 and, consequently, Theorem 3 hold for the other three fractal dimensions as well.

Example 3. Let $G=0.83 \cdots, \phi=1.61 \cdots, e=2.71 \cdots$ and $\pi=3.14 \cdots$ be the Gauss's constant, the golden ratio, the Euler's number and the Pi number, respectively. Then, by Theorem 3 , there are aleph-two thin random virtual fractals with almost surely Hausdorff dimension of $G, \phi, e$ and $\pi$ in $\mathbb{R}^{n \geq 1}, \mathbb{R}^{n \geq 2}, \mathbb{R}^{n \geq 3}$ and $\mathbb{R}^{n \geq 4}$, respectively.

As in the deterministic case, the assertion in Theorem 3 and its proof methodology have two immediate important corollaries: First, the cardinality of the set of random fractals in $\mathbb{R}^{n}$ is aleph-two. Second, the cardinality of the set of random non-fractals in $\mathbb{R}^{n}$ is aleph-two as well. Both results are proved similar to the determinist case [24] and yield from two applications of Cantor-Schroder-Bernstein theorem [39], respectively.

\section{Discussion}

\subsection{Summary}

This work presented an existence theorem for random fractals of a given Hausdorff dimension and a Lebesgue measure with the highest possible cardinal number of alephtwo. In addition, it generalized the former deterministic existence theorem in terms of the randomness and the number of fractal dimensions. It focuses mainly on the generalization process from GHDT to SGHDT. It accomplished this by implementing a special stochastic feature on the construction process of deterministic fat fractals used in establishing GHDT; then, it uses set theoric techniques to upgrade the existential cardinality from continuum to aleph-two. Finally, it uses the equality of four fractal dimensions for the building blocks of the SGHDT in the generalization. To compare the generalization process from HDT to GHDT and from GHDT to SGHDT, we summarize these results as follows.

HDT For any real $r>0$, there is a continuum of [thin deterministic] fractals with Hausdorff dimension $r$ in $\mathbb{R}^{n}(\lceil r\rceil \leq n)$.

GHDT For any real $r>0$ and $l \geq 0$, there are aleph-two deterministic fractals with the Hausdorff dimension $r .1_{\{0\}}(l)+n \cdot 1_{(0, \infty)}(l)$ and Lebesgue measure $l$ in $\mathbb{R}^{n}$ $(\lceil r\rceil \leq n)$.

SGHDT For any real $r>0$ and $l \geq 0$, there are aleph-two random fractals with the fractal dimension $r .1_{\{0\}}(l)+n \cdot 1_{(0, \infty)}(l)$ almost surely, and expected Lebesgue measure $l$ in $\mathbb{R}^{n}(\lceil r\rceil \leq n)$. Here, the fractal dimension is one of four dimensions: Hausdorff dimension, packing dimension, Assouad dimension, box dimension.

Figure 1 presents the generalization process upon comparing the above three statements. 


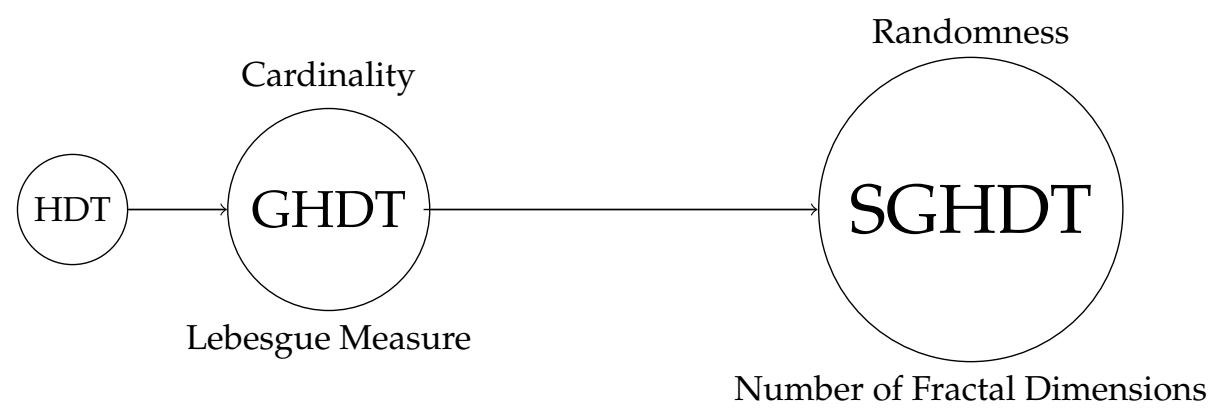

Figure 1. The generalization process of the Hausdorff Dimension Theorem(HDT) in terms of cardinality, Lebesgue measure, randomness and number of fractal dimensions.

\subsection{Contributions}

This work contributes to the fractal geometry literature in four perspectives: First, as in the deterministic case, it highlights the advantages of random Cantor fractals over other conventional random fractals on establishing existential results for random fractals. Second, as in the deterministic case, the main result is equipped with constructive proof rather than pure existential proof. Third, it presents two more sets with the cardinality of aleph-two. Finally, it partially answers open problems \#1 and \#3 in [24]. For the case of open problem \#1, the dimension of the set of distinctive random fractals in $\mathbb{R}^{n}$ is the same for four fractal dimensions considered in this work. Moreover, for the case of open problem \#3, the cardinality of the set of all distinctive fractals in $\mathbb{R}^{n}$ is independent of their deterministic or random nature. We summarize these key results as follows.

Conclusion C1 The cardinality of the set of all distinctive fractals in $\mathbb{R}^{n}$ is invariant regardless of the used fractal dimension.

Conclusion C3 The cardinality of the set of all distinctive fractals in $\mathbb{R}^{n}$ is invariant regardless of their deterministic or random nature.

\subsection{Future Work}

Future work may expand and assess the current results in two directions: First, only four fractal dimensions were considered in SGHDT. It is plausible to evaluate the results for other fractal dimensions, such as the Lyponov dimension [40], the Highuchi dimension [41], the correlation dimension [42] and the information dimension [43]. Second, this work focused on n-dimensional Eulidean spaces as relatively tangible case of fractal settings. It is plausible to establish and evaluate these results for more abstract settings [44].

\section{Conclusions}

This work has presented another partial solution to the problem of retrospective existence of any set of fractals for a given fractal dimension and the Lebesgue measure for the case of random fractals. It presented the case for random fractals with cardinality of aleph-two, and expanded the former deterministic result in terms of randomness and the number of involved fractals dimensions. Finally, it cleared the way for working through the problem for the case of more generalized abstract fractal structure and dimension.

Funding: This research received no external funding.

Institutional Review Board Statement: Not applicable.

Informed Consent Statement: Not applicable.

Data Availability Statement: Not applicable.

Acknowledgments: The author is grateful to the journal reviewers for their constructive comments and suggestions on the first draft of the manuscript.

Conflicts of Interest: The author declares no conflict of interest. 


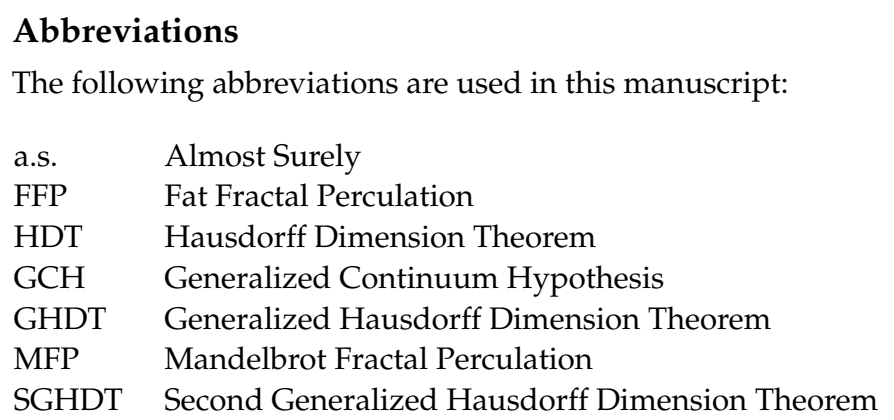

\section{References}

1. Mandelbrot, B.B. Renewal sets and random cutouts. Z. Wahrscheinlichkeitstheorie Und Verwandte Geb. 1972, 22, 145-157. [CrossRef]

2. Mandelbrot, B.B. Intermittent turbulence in self-similar cascades: Divergence of high moments and dimension of the carrier. J. Fluid Mech. 1974, 62, 331-358. [CrossRef]

3. Taylor, S.J. The measure theory of random fractals. Math. Proc. Camb. Philos. Soc. 1986, 100, 383-406. [CrossRef]

4. Falconer, K.J. Random Fractals. Math. Proc. Camb. Phil. Soc. 1986, 100, 559-582. [CrossRef]

5. Graf, S. Statistically self-similar fractals. Probab. Theory Relat. Fields 1987, 74, 357-392. [CrossRef]

6. Frezza, M.; Bianchi, S.; Pianese, A. Fractal analysis of market (in)efficiency during the COVID-19. Financ. Res. Lett. 2021, 38. [CrossRef]

7. Sivakumar, C. Stochastic evolution of the universe: A possible dynamical process leading to fractal structures. Pramana-J. Phys. 2018, 90. [CrossRef]

8. Panigraphy, C.; Seal, A.; Mahato, N.K. Fractal dimension of synthesized and natural color images in lab spaces. Pattern Anal. Appl. 2020, 23, 819-836. [CrossRef]

9. Frege, G. Foundations of Arithmetic; Austin, J.L., Translator; Oxford: Blackwell, UK, 1953.

10. Brouwer, L.E.J. Over de Grondslagen der Wiskunde. Ph.D. Thesis, Universiteit van Amsterdam, Amsterdam, The Netherland, 1907.

11. Waaldijk, F. On the Foundations of Constructive Mathematics-Especially in Relation to the Theory of Continuous Functions. Found. Sci. 2005, 10, 249-324. [CrossRef]

12. Troelstra, A.S.; van Dalen, D. Constructivism in Mathematics: An Introduction (Two Volumes); Elsevier Science, Amsterdam: North Holland, The Netherland, 1988.

13. Dowek, G. Chapter 8: Constructive Proofs and Algorithms. In Computation, Proof, Machine: Mathematics Enters a New Age; Guillot, P., Roman, M., Eds.; Cambridge University Press: Cambridge, UK, 2015.

14. Klaus, M.; Schuster, P.; Schwichtenberg, H. (Eds.) Proof and Computation: Digitalization in Mathematics, Computer Science, and Philosophy; World Scientific: Hackensack, NJ, USA, 2018; pp. 1-46.

15. Xiao, X.; Chen, H.; Bogdan, P. Deciphering the generating rules and functionalities of complex networks. Sci. Rep. 2021, 11, 22964. [CrossRef]

16. Li, H. Fractal analysis of side channels for breakdown structures in XLPE cable insulation. J. Mater. Sci. Mater. Electron. 2013, 24, 1640-1643. [CrossRef]

17. França, L.G.S.; Vivas Miranda, J.G.; Leite, M.; Sharma, N.K.; Walker, M.C.; Lemieux, L.; Wang, Y. Fractal and Multifractal Properties of Electrographic Recordings of Human Brain Activity: Toward Its Use as a Signal Feature for Machine Learning in Clinical Applications. Front. Physiol. 2018, 9, 1767. [CrossRef]

18. Hu, S.; Cheng, Q.; Wang, L.; Xie, S. Multifractal characterization of urban residential land price in space and time. Appl. Geogr. 2012, 34, 161-170. [CrossRef]

19. Brothers, H.J. Intervallic Scaling in the Bach Cello Suites. Fractals 2009, 17, 537-545. [CrossRef]

20. Sharapov, S.; Sharapov, V. Dimensions of Some Generalized Cantor Sets. Available online: http://classes.yale.edu/fractals/ FracAndDim/cantorDims/CantorDims.html (accessed on 3 April 2006).

21. Soltanifar, M. On A Sequence of Cantor Fractals. Rose Hulman Undergrad. Math. J. 2006, 7, 1-9.

22. Squillace, J. Estimating the fractal dimension of sets determined by nonergodic parameters. Discret. Contin. Dyn. Syst.-A 2017, 37, 5843-5859. [CrossRef]

23. Gryszka, K. Hausdorff dimension is onto. Pr. Kola Mat. Uniw. Ped. W Krak 2019, 5, 13-22.

24. Soltanifar, M. A Generalization of the Hausdorff Dimension Theorem for Deterministic Fractals. Mathematics 2021, 9, 1546. [CrossRef]

25. Chen, C. A Class of Random Cantor Sets. Real Anal. Exch. 2017, 42, 79. [CrossRef]

26. Chen, C. Distribution of random Cantor sets on tubes. Arkiv För Matematik 2016, 54, 39-54. [CrossRef]

27. Mandelbrot, B. B. The Fractal Geometry of Nature; W. H. Freeman and Co.: San Francisco, CA, USA, 1982; ISBN 0-7167-1186-9.

28. Falconer, K.J.; Grimmett, G.R. On the geometry of random cantor sets and fractal percolation. J. Theor. Probab. 1994, 7, 209-210. [CrossRef]

29. Rosenthal, J.S. A First Look at Rigorous Probability Theory; World Scientific: Singapore, 2006; pp. 10, 21-22.

30. Koivusalo, H. Dimension of Uniformly Random Self-Similar Fractals. Real Anal. Exch. 2014, 39, 73. [CrossRef] 
31. Broman, E.I.; Van De Brug, T.; Camia, F.; Joosten, M.; Meester, R: Fat fractal percolation and k-fractal percolation. ALEA Lat. Am. J. Probab. Math. Stat. 2012, 9, 279-301.

32. Bishop, C.J. Fractals in Probability and Analysis, 1st ed.; Cambridge Studies in Advanced Mathematics, Series Number 162; Cambridge University Press: Cambridge, UK, 2017; pp. 105-107.

33. Orzechowski, M.E. Percolation in Random Cantor Sets. Fractals 1997, 5, 101-109. [CrossRef]

34. Tricot, C., Jr. Two definitions of fractional dimension. Math. Proc. Camb. Philos. Soc. 1982, 91, 57-74. [CrossRef]

35. Assouad, P. Étude d'une dimension métrique liée à la possibilité de plongements dans Rn. Comptes Rendus de l'Académie des Sciences Série A-B 1979, 288, A731-A734.

36. Bouligand, M.G. Ensembles impropres et nombredimensionnel. Bull. Des Sci. MathéMatiques 1928 52, $320-344$.

37. Schroeder, M. Fractals, Chaos, Power Laws: Minutes from an Infinite Paradise; W. H. Freeman: New York, NY, USA, $1991 ;$ pp. $41-45$.

38. Schleicher, D. Hausdorff Dimension, Its Properties, and Its Surprises. Am. Math. Mon. 2007, 114, 509-528. [CrossRef]

39. Bajnok, B. An Invitation to Abstract Mathematics, 2nd ed.; Springer: Berlin/Heidelberg, Germany, 2020; p. 325.

40. Frederickson, F.; Kaplan, J.; Yorke, E.; Yorke, J. The Liapunov dimension of strange attractors. J. Differ. Equ. 1983, $49,185-207$. [CrossRef]

41. Higuchi, T. Approach to an irregular time-series on the basis of the fractal theory. Phys. D 1988, 31, 277-283. [CrossRef]

42. Peter, G. Generalized Dimensions of Strange Attractors. Phys. Lett. A 1983, 97, 227-230.

43. Rényi, A. On the dimension and entropy of probability distributions. Acta Math. Acad. Sci. Hung. 1959, 10, 193-215. [CrossRef]

44. Fernández-Martínez, M.; Sánchez-Granero, M. Fractal dimension for fractal structures. Topol. Its Appl. 2014, 163, 93-111. [CrossRef] 